factors for changes in exposed numbers and levels are applied in estimation intervals within the REPs.

Results The methods are illustrated using a range of scenarios for reducing lung cancer due to occupational exposure to respirable crystalline silica (RCS). AFs for lung cancer due to RCS could potentially be reduced from $2.07 \%$ in 2010 to nearly zero by 2060 , depending on the timing and success of interventions.

Conclusion The importance of focusing on achieving compliance with current exposure standards in small industries is highlighted and can be more effective than setting standards at a lower level. The method can be used to highlight high-risk carcinogens, industries and occupations and is designed to allow comparative estimates to be made even where exposure data are limited. It is adaptable for other countries and other exposure situations in the general environment and can be extended to include socio-economic impact assessment.

\section{P1-176 BIAS ESTIMATION METHODS FOR THE BURDEN OF OCCUPATIONAL CANCER}

doi:10.1136/jech.2011.142976d.69

S Hutchings, ${ }^{*}$ L Rushton. Imperial College London, London, UK

Introduction Bias occurs in particular when estimates are based on sparse or inadequate data. We have estimated the burden of cancer in Great Britain attributable to occupation using an attributable fraction (AF) methodology, and present an adaptation of Greenland's ${ }^{1}$ Monte-Carlo sensitivity analysis (MCSA) to account for bias uncertainty.

Methods Sources of bias in burden estimation include using Levin's estimator with adjusted RR, unknown cancer latency, unknown proportions exposed and inadequate estimates of employment turnover. Each source of bias operates on a component of the AF estimator, which is represented by a factor for which a prior distribution is estimated from independent sources. Monte-Carlo repeated sampling from these distributions is then used, recalculating the AF each time.

Results Results are presented graphically for a hierarchy of bias sources that contribute to an overall credibility interval for the AF. For sinonasal cancer and wood dust the intervals for bias due to the variables contributing to the proportions exposed are narrower than the interval for $\mathrm{RR}$ random error only, and bias from incorrect use of Levin's estimator makes the least contribution.

Conclusion The method presented illustrates the use of credibility intervals to indicate important sources of uncertainty and facilitates identification of data gaps and future research needs.

\section{REFERENCES}

1. Greenland S. Interval estimation by simulation as an alternative to and extension of confidence intervals. Int J Epidemiol 2004;33:1389-97.

2. Greenland S. Multiple-bias modelling for analysis of observational data. J Royal Statist Soc Series A 2005;168(Part 2):267-306.

\section{P1-177 DEVELOPMENT AND EVALUATION OF A FORECASTING MODEL FOR INFECTIOUS DISEASES IN JAPAN USING TIME-SERIES ANALYSIS}

doi:10.1136/jech.2011.142976d.70

${ }^{1} \mathrm{M}$ Inoue, ${ }^{*} \mathrm{~S}$ Hasegawa, ${ }^{3} \mathrm{~A}$ Suyama. ${ }^{1}$ Tottori University, Yonago, Japan; ${ }^{2}$ Hokkaido Institute of Public Health, Sapporo, Japan; ${ }^{3}$ Radiation Effects Research Foundation, Nagasaki, Japan

Having forecasts for infectious diseases can help support risk management and effective intervention against the outbreak of disease. The main object of this study was to obtain a suitable way of making predictions of disease incidents through the use of timeseries analysis.

Methods Retrospective data of Japanese infectious diseases were collected for the period of 2000-2010. In this study, influenza, mumps and infectious gastroenteritis were used for analysis. These data were separated into two groups: one (2000-2007) was designated the "training" set and the other (2008-2010), the "validation" set. We applied three models: an exponential smoothing method (ESM), an autoregressive integrated moving average (ARIMA) and a nearest neighbour method (NNM), to make predictions on the morbidity of the diseases. Statistical analysis for the ESM and the ARIMA model were carried out using SPSS Ver.19. The NNM was executed using a computer program made by us based on its' algorithm. We used the mean absolute percentage error (MAPE) to measure and quantify how well the data matched or "fit". For example, a lower MAPE value would indicate a better fit of the data. Results The best-fit model for influenza was the NNM, where the MAPE was $70 \%$. For mumps and infectious gastroenteritis, the ARIMA revealed the best fit, where the MAPE for these were $7 \%$ and $16 \%$, respectively.

Conclusion We found that the ARIMA and NNM provide a useful way of making predictions of disease. The models could well be used in planning for risk management against infectious diseases.

\section{P1-178 HETEROGENEITY OF THE 23-VALENT PNEUMOCOCCAL POLYSACCHARIDE VACCINE EFFICACY DUE TO VARIOUS UNDERLYING CONDITIONS OF CHRONIC PULMONARY DISEASE IN ELDERLY PATIENTS}

doi:10.1136/jech.2011.142976d.71

${ }^{1} \mathrm{~S}$ Inoue, ${ }^{2} \mathrm{~T}$ Kaneko, ${ }^{2} \mathrm{~S}$ Morita, ${ }^{3} \mathrm{Y}$ Natsumeda, ${ }^{1} \mathrm{~S}$ Mizushima. ${ }^{1}$ Yokohama City University, Graduate School of Medicine, Department of Epidemiology and Public Health, Yokohama, Japan, ${ }^{2}$ Yokohama City University, Graduate School of Medicine, Department of Biostatistics and Epidemiology, Yokohama, Japan; ${ }^{3}$ Yokohama City University, Graduate School of Medicine, Laboratory for Clinical Research, Yokohama, Japan

Introduction The efficacy of 23-valent pneumococcal polysaccharide vaccine (PPV23) against pneumonia in elderly with chronic illness remains controversial.

Methods A total of 1378 elderly outpatients with chronic pulmonary disease were informed of the PPV23 efficacy on infectious pulmonary exacerbations. Individual patients who responded affirmatively were vaccinated between August and November 2002 and rest of them was assigned for control. The prevaccine period was defined as August 2001-August 2002. The events of interest were the first episode of bacterial pulmonary infection and death from any cause. The participants were observed for 2 years after vaccination.

Results Frequent episodes of pulmonary infection in the prevaccine period increased the event rate significantly (no episode $17.3 \%$, one episode $48.3 \%$, more than one episode $74.6 \%$, $p<0.001$, the KaplanMeier method). No significant differences of the survival probability were observed between the vaccinated and unvaccinated group during the analysis of the whole cohort. The mortality rate was higher in the vaccinated group $(p<0.010)$. This was due to the higher vaccination rate in patients who had pulmonary infection during the prevaccine period $(p<0.001)$. No harmful effects of PPV23 on mortality rate was seen $(p=0.100)$ in the COX proportional hazards regression model. In subgroup analysis, PPV23 reduced event rate significantly only in patients with chronic respiratory failure who had not pulmonary infection during the prevaccine period $(p<0.019)$

Conclusion The PPV23 needs to be given to elderly patients with chronic pulmonary disease at an earlier time where infectious complication in the lung has not occurred yet. 